\title{
Abstract Author Index
}

by abstract number

A

Adams, C.A., P25

Adams, Jr., C., P21, P35

Ahmed, N., P20

Alagiakrishnan, K., P19

Alizo Arruebarrena, G., P43

Alverdy, J., O02, O03, O12, O13, O30, P36, P38

Amatullah, H., P12

An, G.C., P39

Anderson, A., P45

Anderson, K., O37

Andrews, A., P27

Apostolides, G., P64

Armand, P., P24

Armstrong, C., O24

Arron, M., O03, O30

Askari, R., P15, P24, P69

Ayala, A., O40, P35

\section{B}

Badal, R., P40

Barie, P., O19, P05

Barnard, D., P01

Becker, P., P56

Begier, E., P45, P48

Beilman, G., O14, O33, P63

Bell, B., O39

Bellister, S., P55

Belogortseva, N., O30, P38

Benjamin, A., P39

Benns, M., P31

Benoit, E., P21

Bickler, S., O41, P13

Billiar, T., O10

Bindokas, V., O02

Blackwood, B., O38, P22

Bochicchio, G., O35, P57

Bonatti, H., P23, P46, P62

Bonne, S., O15

Borscheid, R., P69

Bosslet, G., P29

Bouchillon, S., P40

Bowling, B., O09

Bowling, J., O09, O39

Boyle-Vavra, S., O13

Bradburn, E., O26

Bradley, J., P27

Brady, J., P66

Brajcich, B., O20

Brakenridge, S., O25, P61
Branski, L., P49

Brat, G., P15

Brathwaite, C., P54

Broach, T., P29

Bruns, B., P32

Buchanan, L., P67

Buckman, S., O35, P03

Bulger, E., O15

Buzogany, I., P34

C

Caldarone, C., O05

Cammarata, S., O23

Cauvi, D., O41, P13

Celestin, A., O42

Chaffin, A., P53

Chamieh, J., O27, P57

Chang, R., O18

Chen, H., P32

Chen, Q., P51

Cheronis, J., P42

Chiu, W., P67

Cho, K., P10

Christley, S., O12

Chun, T., O40, P35

Churchill, T., P19

Cioffi, W., O40, P21, P25, P35

Claridge, J., O17, O20

Clatterbuck, B., P47

Clayton, R., P49

Clement, L.P., P01, P09

Coimbra, R., O06

Cole, M., P49

Coleman, M., P24

Coleman, N., P24

Collier, B., O26

Collier, Z., P36

Cook, C., O42

Cook, J., P37

Cooper, D., P45

Cooper, Z., P24

Costantini, T., O06

Cotton, B.A., O18

Crews, H., P29

Croce, M., P01, P09

Croft, C., O25, P61

D

D'Cruz, R., P12

Danner, R., O15

Darwiche, S., O36
Davenport, K., P27

Davies, S., P26

Davis, C., P57

Davis, J., P43, P47

De Maio, A., O41, P13

De Raet, J., P60

DeFazio, J., O02

Deng, M., O10

Dhiman, N., O26

Diaz, Jr., J., P32

Dickinson, C., P25

Dickinson, L., P43

Dickinson, S., P28

Diebel, L., O32

Dietch, Z., P26

Dolan, J., O21, P15

Domjan, Z., P34

Dos Santos, C., P12

Dosokey, E., P66

Dossa, A., O39, P14

Drew, L., P54

Duane, T., O44

\section{E}

Earl-Royal, E., O19

Efem, R., P54

Efron, P., O25, P61

Eiden, J., P45

Eley, A., P10

Eliceiri, B., O06

Elmalaki Hossain, L., P16, P44

El-Shafy, I.A., P65

Elwood, N., O43

Escobar, O., O09, P14

Evans, C., P01

Evans, H., O15, O24, P58, P59

F

Fabian, T., P01, P09

Fairbanks, T., P27

Faist, E., P17

Falimirski, M., P29

Fallon, E., O40, P35

Farmer, D., O44

Finnerty, C., P49, P50

Firek, B., P52

Fisher-Stucky, E., P27

Flores-Gonzalez, J., O34

Folkerson, L., O18

Follman, D., O15

Ford, H., O09, O39, P41 
Franklin, G.A., P31

Fraser, T., O04

Friedman, C., P28

Fuchs, A., P57

Fujimi, S., P07

\section{G}

Galbraith, N., P11

Gardner, S., P11

Gayer, C.P., O09, P14

Gessner, B., P59

Gilbert, J., O12

Giuca, M., P54

Glover, J.J., O14, O33, P63

Golden, J., O09, O39

Goldman, J., P37

Golob, Jr., J., O17

Gorgun, E., O04

Gornicsar, K., P34

Grabowski, J., O38

Grasa González, F., P16, P44

Green, T., P10

Greenhalgh, D., O07, P10

Grishin, A., O09, O39, P41

Grosz, A., P34

Gruber, W., P45

Gu, G., P68

Guidry, C., O01, O22, O43,

O45, P04, P26

Gupta, S., P12

Gurtman, A., P45

Guyton, K., O12, O13, O30

H

Haac, B., P30

Hadley, A., O11

Halbach, J., O41, P13

Hamill, M., O26

Harbrecht, B., P31

Harris, A., P30

Harris, J., P22

Harter, C., O30

Hartzler, A., O24

Harvin, J., O18

Hassinger, T., O45

Havens, J., P24

Hawishir, D., P13

He, J., O20

Heffernan, D., O40, P21, P25, P35

Hemalkumar, M., P50

Hennessy, L., O15

Henry, E., O23

Henry, S., P67

Herndon, D., P49, P50

Herrera, A., P32

Hershberger, E., O29

Hilfiker, M., P27

Ho, V., P66

Hoffman-Roberts, H., P05

Hohmann, S., O15

Holcomb, J., O08, O18, P06, P08

Holena, D., O19

Holihan, J., O34
Holroyd-Leduc, J., P19

Hooper, D., O15

Hopkins, M., P43

Horkan, D., O31

Horn, P., P05

Hossain, L.E., P16

Hranjec, T., O22

Hubert, N., O12

Hunter, C., P22

Hyoju, S., O02, O03, O13

\section{I}

Illingworth, L., O09

Isani, M., O09, O39, P41

Ishikawa, S., O21

Itani, K., P51

Ito, H., P07

\section{J}

Jabir, M., P66

Jansen, D., P53

Jansen, K., P45

Jia, S.-H., P12

Johnson, D., P62

Johnson, J., P36

Jordan, J., O25, P61

Joseph, S., P37

K

Kadri, S., O15

Kale, A., P55

Kao, L., O34, O37, P55

Kaplan, L., P33

Karcutskie, C., O31

Kaufman, E., O19

Kavarian, P., O09

Kawamura, A., P07

Keegan, J., O21, P15

Khadaroo, R., P19

Khairat, A., P62

Kheirbek, T., P21, P25

Kiguchi, T., P07

Kimbrough, C., P31

Kingsley, J., O23

Kinoshita, T., P07

Klabbers, R., O03

Kline, S., O33

Ko, T., O34, P55

Kolesnik, O., P67

Kooker, P., P20

Kraft, R., P17

Krezalek, M., O02, O03, O12, O13, P38

Kubo, N., P07

Kuo, Y.H., P47

L

Lally, K., O37

Langness, S., O06, O41, P27

Lauerman, M., P32, P67

Lawrence, L., O23

Lederer, J., O21, P15

Lee, E., O28

Lee, J.O., P49, P50
Lemon, S., O25

Lentini, P., P42

Lenz, A., P17

Leung, C.H., O05

Leventhal, S., P10

Levine, Z., O12, O30

Lewis, A., O16, O28

Li, J., P68

Li, Y., P68

Li, Y.T., P19

Liang, M., O34

Liberati, D., O32

Lim, D., P10

Lim, J., O09

Lin, K., P48

Lipskar, A., P65

Liu, S., P68

Lober, W., O24

Loftus, T., O25, P61

Lollar, D., O26

Long, J., O29

Loor, M., O14, P63

Lordon, R., O24

Louras, P., O15

Lourd, M., P56

Love, K., P31

\section{M}

Macaluso, A., P08

Mackowski, M., P31

Magnotti, L.J., P01, P09

Mallicote, M., P14

Maloney, C., P65

Marino, N., P27

Marsh, A., P05

Marshall, J., P12

Martin, N., P33

Mateo, R., P46

Matsuda, H., P07

Matthew, N., O10

Mayampurath, A., P36

Mazuski, J., O29, O35, P03

McConnell, K., O11

McIntyre, A., P47

Meizoso, J., O31

Messier, R., P47

Metcalfe, D., P69

Millas, S., P55

Miller, B., O29

Miller, K., P31

Mohr, A., O25, P61

Monaghan, S., P21, P25

Moore, B., O44

Moore, F., O25, P61

Moore, L., O08

Morancy, J.D., O14, P63

Morowitz, M., P52

Mózes, T., P34

Mueck, K., O37, P55

Muratore, C., O40

Muratore, S., O33 
N

Nakahori, Y., P07

Nakamoto, N., P07

Namias, N., O31

Napolitano, L., P28

Neal, M., P52

Nguyen, L., O25

Nohra, E., P57

Nomura, J., P48

Nunez Lopez, O., P49, P50

Nunez, H., P21, P25

O

O'Riordan, W., O23

Odom, S., O42

Okafor, E., O13

Olufajo, O., P69

Oriel, B., P51

Ott, R., P63

Ozben, V., O04

$\mathbf{P}$

Padwal, R., P19

Palmieri, T., O07

Park, H., P67

Park, P., P28

Pascual, J., P33

Patil, P., P28

Patton, M., P45

Pelczar, A., P62

Peter, A., P37

Pineles, L., P30

Pinnola, A., P47

Polk, H., P11

Popejoy, M., O29

Prince, J., P65

Proctor, K., O31

Putnam, L., O37

Q

Quintas, M., O23

$\mathbf{R}$

Radhakrishnan, H., P06

Raghavendran, K., P28

Ramonell, K., O11

Rashid, N., P48

Ray, J., O31

Remes, J., P60

Remzi, F., O04

Ren, J., P18, P68

Rencuzogullari, A., O04

Reynolds, H., P66

Reynolds, N., P42

Rhee, C., O15

Rimal, R., O26

Rios-Diaz, A.J., P69

Robu, M., P60

Rock, C., P30

Rodriguez Ramos, M., P16

Rogers, M., P52

Romano, A., P43
Rosenberger, L., O01

Rosengart, M., O16, O28

Roth, J., P56

Rotstein, O., O05

Ruiz, X., O31

S

Sahm, D., P40

Salim, A., P15, P24, P69

Sanchez Relinque, D., P44

Sanders, J., O44

Sanger, P., O24

Savakus, J., O20

Sawyer, R., O01, O22, O42, O43, O45, P03, P04, P26

Scalea, T., P67

Scerbo, M., P06, P08

Schechtman, D., O17, O20

Schrick, E., P58, P59

Schroeppel, T., P01, P09

Schulman, C., O31

Sciarretta, J., P43, P47

Scoble, P., P05

Scott, M., O10

Seiden, D., P45

Sen, S., O07

Seshadri, A., O21, P15

Severs, J., P45

Seymour, C., O16

Shah, Puja, O01, P26

Shakhsheer, B., O30

Sharpe, J., P09

Shimizu, K., O21

Sim, J., P21

Simianu, V., O24

Simons, M., P42

Smith, J., P31

Smith, S., O25, P61

Solomkin, J., O29

Statz, C., O14, P63

Steele, S., P66

Stein, D., P30, P67

Stein, S., P66

Stephen, A., P21, P25, P35

Stocchi, L., O04

Strong, A., P53

Suffredini, A., O15

Swanson, J., P01

Swartz, M., O08, O18

Swihart, B., O15

Symons, W., O27

$\mathbf{T}$

Tarras, S., O32

Tesoriero, R., P32, P67

Tessier, J., O44

Theodorou, C., P22

Thom, K., P30

To, K., P28
Totten, C., P56

Trentzsch, H., P17

Tsang, J., P12

Tsao, K., O37

Tseng, C., O23

Turnbull, I., O35, P03, P57

V

Vasconcellos, K., P64

Versten, L., O12, O30

Villegas, N., O08

Voigt, C., P49, P50

Voils, S., O25

W

Wade, C., O08, O18

Wagg, A., P19

Walker, P., P33

Walker, S., P11

Waltz, P., O36

Wang, G., P68

Wang, J., O39

Wang, Y., O36

Wanke-Jellinek, L., O21

Ward, E., O41

Warkentin, L., P19

Weinberg, J.A., P01, P09

Weinert, C., O33

Wen, Y., P66

Wert, Y., P37

Wiest, C., P37

Winfield, R., P57

Woelber, E., P58, P59

Wojahn, A., O20

Wolfe, E., P53

Wong, K., P51

Wood, G.C., P01

Wray, C., P55

Wu, Q., P18

Wu, X., P68

Wurzer, P., P49, P50

$\mathbf{Y}$

Yamakawa, K., O21

Yeh, A., P52

Yorkgitis, B., P15, P69

Yoshikawa, Y., P07

Yu, H., P48

Yuan, D., O16, O28

$\mathbf{Z}$

Zaborin, A., $\mathrm{O} 02$

Zaborina, O., O02, O03, O12, O13, O30, P38

Zedler, S., P17

Zhang, X., O16, O28

Zhou, H., O10

Zito, E., P45

Zosa, B., O17

Zuckerbraun, B., O28, O36, P52 